\title{
A novel ABCG-like transporter of Trypanosoma cruzi is involved in natural resistance to benznidazole
}

\author{
Bianca Zingales ${ }^{1 /+}$, Rafael Gomes Aquino Araujo', Margoth Moreno', Jaques Franco', \\ Pedro Henrique Nascimento Aguiar' ${ }^{2}$, Solange Lessa Nunes', Marcelo Nunes Silva', \\ Susan lenne', Carlos Renato Machado², Adeilton Brandão ${ }^{3}$
}

\author{
'Departamento de Bioquímica, Instituto de Química, Universidade de São Paulo, São Paulo, SP, Brasil \\ ${ }^{2}$ Departamento de Bioquímica e Imunologia, Instituto de Ciências Biológicas, Universidade Federal de Minas Gerais, Belo Horizonte, MG, Brasil \\ ${ }^{3}$ Laboratório Interdisciplinar de Pesquisas Médicas, Instituto Oswaldo Cruz-Fiocruz, Rio de Janeiro, RJ, Brasil
}

Benznidazole (BZ) is one of the two drugs used for Chagas disease treatment. Nevertheless therapeutic failures of BZ have been reported, which were mostly attributed to variable drug susceptibility among Trypanosoma cruzi strains. ATP-binding cassette $(A B C)$ transporters are involved in a variety of translocation processes and some members have been implicated in drug resistance. Here we report the characterisation of the first $\mathrm{T}$. cruzi $A B C G$ transporter gene, named TcABCG1, which is over-expressed in parasite strains naturally resistant to BZ. Comparison of TcABCG1 gene sequence of two TcI BZ-resistant strains with CL Brener BZ-susceptible strain showed several single nucleotide polymorphisms, which determined 11 amino acid changes. CL Brener transfected with TcI transporter genes showed 40-47\% increased resistance to BZ, whereas no statistical significant increment in drug resistance was observed when CL Brener was transfected with the homologous gene. Only in the parasites transfected with TcI genes there was 2-2.6-fold increased abundance of TcABCG1 transporter protein. The analysis in wild type strains also suggests that the level of TcABCG1 transporter is related to BZ natural resistance. The characteristics of untranslated regions of TcABCG1 genes of BZ-susceptible and resistant strains were investigated by computational tools.

Key words: Chagas disease - benznidazole resistance - ABCG transporter - T. cruzi strains - transcript abundance - single nucleotide polymorphisms

The protozoan Trypanosoma cruzi is the etiological agent of Chagas disease, which ranks among the world's most neglected diseases. According to estimates by the Pan American Health Organization and the World Health Organization (WHO), 7.7-10 million people are chronically infected with $T$. cruzi and 10,000-14,000 deaths per year are caused by Chagas disease (Moncayo \& Silveira 2009, WHO 2012). Only two drugs are available for Chagas disease treatment, benznidazole (BZ) and nifurtimox (NF). Both drugs are nitroheterocyclic compounds which frequently cause severe side effects and have limited efficacy in the chronic phase of the disease (Rassi Jr et al. 2012). The reasons for treatment failures are unknown, but may be due to variable drug susceptibility among $T$. cruzi strains, characteristics of the host's immune system and/or unfavourable drug pharmacokinetic properties (Urbina 2010). Nevertheless, several nonrandomised clinical studies report the efficacy of the etiological treatment to reduce the progression of chronic Chagas heart disease (Sosa-Estani et al. 2009).

doi: $10.1590 / 0074-02760140407$

Financial support: FAPESP (2013/13333-8), CNPq (304793/2009-4) (to BZ), FAPEMIG (APQ-02308-13) (to CRM), PROEP/IOC/ FIOCRUZ/CNPq (400124/2011-4) (to AB)

+Corresponding author: bszodnas@iq.usp.br

Received 31 October 2014

Accepted 5 March 2015
Although in recent years novel chemical classes have shown promising activity against $T$. cruzi, no new drug is under development (Zingales et al. 2014). Therefore, efforts should be devoted to elucidating mechanism(s) underlying BZ and/or NF natural resistance.

Experimental evidence from several laboratories has shown that $\mathrm{BZ}$ and NF exhibit divergent activities against different T. cruzi strains (Filardi \& Brener 1987, Murta et al. 1998, Villarreal et al. 2004, Moreno et al. 2010). Such differences most certainly are related to the high phenotypic and genotypic diversity among T. cruzi strains. Currently the parasite strains are classified into six discrete typing units (DTUs), designated as TcI-TcVI (Zingales et al. 2009). The rationale for this classification, DTU eco-epidemiological features and correlation with natural and experimental infection has been reviewed (Miles et al. 2009, Zingales et al. 2012).

Specific ATP-binding cassette (ABC) transporters have been implicated in drug resistance in protozoan parasites such as Leishmania, Trypanosoma and Plasmodium (Klokouzas et al. 2003, Leprohon et al. 2006, Sauvage et al. 2009). ABC transporters constitute one of the largest families of membrane proteins that mediate the ATP-driven unidirectional transport of a variety of molecules across biological membranes (Higgins 1992). Substrates include lipids, amino acids, peptides, toxins and chemotherapeutic drugs. $\mathrm{ABC}$ transporters have been associated to the development of resistance of tumours to anticancer drugs, as well as antibiotic resistance in pathogenic microorganisms (Klokouzas et al. 2003, Higgins 2007, Sharom 2008, Sauvage et al. 2009). 
In general, $\mathrm{ABC}$ transporters are composed of two hydrophobic transmembrane domains (TMDs), each usually with six alpha-helical transmembrane segments, and two cytoplasmic nucleotide-binding domains (NBDs). The NBDs contain three conserved sequence elements: the Walker A and Walker B motifs and the ABC signature sequence, which is characteristic of $\mathrm{ABC}$ proteins (Higgins 1992). The TMDs form the putative pathway for substrates across the lipid bilayer and are believed to determine the substrate specificity of the transporter (Velamakanni et al. 2007). ABC "full-size" transporters, symbolised as TMD-NBD ${ }_{2}$, are mainly localised in the plasma membrane. Numerous ABC proteins, called "half-size" transporters, are composed of a single NBD fused to a single TMD. According to the $\mathrm{N}$ or C-terminal location of these domains, they are represented as TMDNBD or NBD-TMD. The "half-size" transporters undergo homo or heterodimerisation for their physiological activity (Velamakanni et al. 2007). Eukaryotic ABC proteins are divided into eight families (ABCA-ABCH) (Igarashi et al. 2004). Representatives of the ABCA, $A B C B$ and $\mathrm{ABCC}$ ("full-size" transporters) and $\mathrm{ABCD}$ and $\mathrm{ABCG}$ ("half-size" transporters) families have been described in protozoan parasites [reviewed by Klokouzas et al. (2003), Leprohon et al. (2006) and Sauvage et al. (2009)].

In the present paper we report the identification of one $\mathrm{ABC}$ transporter gene of the $\mathrm{G}$ family, named $T c A B$ $C G 1$, which is over-expressed in parasite strains naturally resistant to $\mathrm{BZ}$. Transfection of the TCABCG1 gene of two BZ-resistant strains in CL Brener BZ-susceptible epimastigotes increased by $40-47 \%$ the $50 \%$ inhibitory concentration $\left(\mathrm{IC}_{50}\right)$ value to $\mathrm{BZ}$ in the recipient parasites. The relative abundance of TcABCG1 transcripts and the level of the transporter protein were determined in the transfected cultures. The data suggest that $T c \mathrm{AB}-$ CG1 transporter is one of the elements involved in $T$. cruzi resistance to BZ.

\section{MATERIALS AND METHODS}

Parasite strains and cultivation - The characteristics of the T. cruzi strains employed in this study are sum- marised in Table I. Epimastigote forms were cultured in liver infusion-tryptose medium supplemented with $10 \%$ foetal calf serum at $28^{\circ} \mathrm{C}$.

Primers - The sequence and characteristics of the primers are described in Supplementary Table I.

Assay to evaluate BZ susceptibility - The assay was performed as previously reported (Moreno et al. 2010), with exponentially growing epimastigotes. Three independent assays with three replicates in each assay were performed on separate days. For calculation of $\mathrm{IC}_{50}$, corresponding to the drug concentration that inhibited parasite growth by $50 \%$, the data were treated with SigmaPlot v.11 software (Systat Software Inc), employing the four-parameter logistic equation.

DNA microarray experiments - DNA microarray slides, kindly provided by the Pathogen Functional Genomics Resource Center, bearing replicates of 70-mer oligonucleotides representative of 12,288 CL Brener open reading frames (ORFs) (pfgrc.jcvi.org/index.php/ microarray/array_description/trypanosoma_cruzi/version1.html) were employed. Total parasite RNA was extracted with Trizol reagent (Life Technologies) and DNase-treated according to standard procedures. Approximately $20 \mu \mathrm{g}$ RNA was labelled with Cy3 or Cy5dCTP for the first-strand cDNA synthesis (Baptista et al. 2004). Hybridisation conditions, image acquisition and data analysis were as previously described (Baptista et al. 2004, 2006). Data were obtained for two biological replicates (independent parasite harvests). For each replicate a dye-swap experiment was performed.

Real time reverse transcription-polymerase chain reaction (RT-PCR) - RT of $5 \mu \mathrm{g}$ DNAse-treated total RNA was carried out with the SuperScript First-Strand Synthesis System (Invitrogen) and Oligo(dT) $)_{12-18}$ primers, according to the manufacturer's instructions. The levels of TCABCG1 transcripts were determined by real-time PCR using the product of cDNA synthesis reactions as template and ABC.F and ABC.R primers for the TCAB$C G 1$ gene. Real-time PCR was performed in the Mas-

TABLE I

Characteristics of Trypanosoma cruzi strains

\begin{tabular}{|c|c|c|c|c|}
\hline Strain & $\mathrm{DTU}^{a}$ & Host/vector & $\begin{array}{l}\mathrm{IC}_{50} \mathrm{BZ}(\mu \mathrm{M}) \\
(\text { mean } \pm \mathrm{SD})^{b}\end{array}$ & $\begin{array}{c}\text { BZ } \\
\text { phenotype }\end{array}$ \\
\hline Silvio X10 cl1 & TcI & Homo sapiens & $26.1 \pm 2.5$ & $\mathrm{R}$ \\
\hline $\mathrm{YuYu}$ & TcI & Triatoma infestans & $40.5 \pm 1.8$ & $\mathrm{R}$ \\
\hline Colombiana & TcI & H. sapiens & $34.1 \pm 0.8$ & $\mathrm{R}$ \\
\hline Berenice 62 & TcII & H. sapiens & $14.6 \pm 3.6$ & $\mathrm{~S}$ \\
\hline VL 10 & TcII & H. sapiens & $30.4 \pm 2.9$ & $\mathrm{R}$ \\
\hline Esmeraldo cl3 & TcII & H. sapiens & $26.7 \pm 0.3$ & $\mathrm{R}$ \\
\hline 115 & $\mathrm{TcV}$ & H. sapiens & $7.6 \pm 1.6$ & $\mathrm{~S}$ \\
\hline CL Brener & $\mathrm{TcVI}$ & T. infestans & $13.2 \pm 0.8$ & $\mathrm{~S}$ \\
\hline
\end{tabular}

$a$ : discrete typing units (DTUs) classified according to Zingales et al. (2009); $b$ : benznidazole (BZ) sensitivity in epimastigotes; $\mathrm{IC}_{50}: 50 \%$ inhibitory concentration; R: resistant; S: susceptible; SD: standard deviation. 
tercycler Realplex apparatus (Eppendorf) using $\mathrm{SYBR}^{\circledR}$ Green PCR Master Mix (Applied Biosystems). The relative amount of PCR products was determined based on the threshold cycle value and amplification efficiencies and was normalised by dividing the values by the relative amount of the glyceraldehyde 3-phosphate dehydrogenase $(G A P D H)$ gene (XM_814806) used as calibrator and amplified with the pair of primers GAPDH.F and GAPDH.R. All the samples were tested in triplicates in two independent experiments.

Cloning and sequencing of TcABCG1 gene - TcAB$C G 1$ gene of the YuYu strain was PCR amplified from total DNA with the pair of primers TcABCG1.For and $T c$ ABCG1.Rev flanking the gene ORF. Amplicons were cloned into $\mathrm{pGEM}^{\circledR}$-T easy vector (Promega). At least six plasmid clones were sequenced. Chromatograms were analysed with Phred-Phrap-Consed software (Phred scores > 80) (Gordon et al. 1998). The processed sequences were clustered generating the consensus sequence. Nucleotide and predicted amino acid sequences were aligned using CLUSTALX 2.0.12 and alignments were manually adjusted using BioEdit.

Transfection studies - The complete TcABCG1 ORF of CL Brener, Silvio and YuYu strains was PCR-amplified from genomic DNA with the proofreading $P f u$ DNA polymerase (Fermentas) and the pair of primers $T c A B-$ CG1.For and TcABCG1.Rev. Amplicons were cloned in pGEM-T Easy vector (Promega) and the recombinant plasmids were recovered by alkaline lysis using the Wizard Plus SV Miniprep kit (Promega). The identity of the cloned genes was confirmed by sequencing. The expression vector pROCKNeo (DaRocha et al. 2004) was used in the transfection studies. This vector allows the integration of the foreign gene in the $\beta$-tubulin $l o$ cus of T. cruzi (DaRocha et al. 2004). TcABCG1 gene cloned in pGEM-T Easy was released from the vector by digestion with $X b a \mathrm{I}$ and $X h o \mathrm{I}$ enzymes and ligated to pROCKNeo digested with the same enzymes. CL Brener epimastigotes were transfected by electroporation with $50 \mu \mathrm{g}$ linearised plasmid DNA digested with NotI as previously described (DaRocha et al. 2004). Transfected parasites were selected after eight weeks culturing in the presence of $200 \mu \mathrm{g} / \mathrm{mL}$ neomycin.

Southern blot - Genomic DNA was digested with BamHI, separated on $0.8 \%$ agarose and analysed by probing with $\alpha_{-}^{-32} \mathrm{P}$ radiolabelled probes. A 616-base pairs (bp) DNA fragment localised between nt 911-1527 of the TCABCG1 gene was independently amplified from CL Brener and Silvio genomic DNA with the pair of primers TcABCG616F and TcABCG616R. A 435-bp probe for the neomycin resistance $(N e o R)$ gene was amplified from pROCKNeo DNA with the pair of primers Neo-F and Neo-R. Blotting and generation of radioactive DNA probe fragments by random primed labelling were performed as described (Baptista et al. 2006).

Antibodies to TCABCG1 and immunoblotting - A region comprised between nt 232-960 of the TCABCG1 gene was amplified by PCR with PQE2.F and PQE2.R primers and cloned in the expression vector pQE-30 Xa (Quiagen). After transformation of Escherichia coli XL1 Blue MR, standard procedures were followed for the induction and purification of the $\sim 26 \mathrm{kDa}$ recombinant protein. Antibodies were obtained in male Balb/c mice following immunisation with the recombinant protein by intraperitoneal route. Western blot was performed with total protein extract $\left(2 \times 10^{7}\right.$ cells/lane) resolved on $8 \%$ sodium dodecyl sulfate polyacrylamide gel electrophoresis. Proteins were electrotransferred to midi nitrocellulose membranes with a Trans-Blot Turbo Transfer Starter System (BioRad) and incubated with the antibodies at $4^{\circ} \mathrm{C}$ for $16 \mathrm{~h}$. After washing, bound antibodies were detected with the secondary antibody goat anti-mouse IgG, conjugated to IRDye 800 $\mathrm{CW}$ (Li-COR, GmbH, Germany) at the dilution 1:15,000 in tris buffered saline-Tween $0.05 \%$ for $1 \mathrm{~h}$ at room temperature. To assess variations in protein loading, the membrane was incubated with a polyclonal antibody to T. cruzi glycosomal GAPDH (gGAPDH) and processed with a second antibody goat anti-rabbit IgG as above. The images were obtained with LiCOR Odyssey Infared Scanner and analysed with Image Studio 4.0 software.

In silico analysis of the 5' and 3' untranslated regions (UTR) of TcABCG1 genes - To estimate the position of the 5'UTR, we used an approach that takes into account two parameters: (i) the length of 5'UTR for any T. cruzi gene does not exceed $20 \%$ of the respective ORF length (Brandão \& Jiang 2009) and (ii) the relative position of poly-pyrimidine tracts preceding a dinucleotide $\mathrm{AG}$ (possible trans-splicing site) in a segment upstream of initiation codon (Campos et al. 2008). We estimated 3'UTRs based upon previous work of Brandão and Jiang (2009), who pointed out that 3'UTR is on average 3-3.5 x longer than 5'UTR. For the sequences of the genes of Esmeraldo, Silvio, CL Brener NEsmo and Esmo haplotypes, we estimated a 5'UTR length of $\sim 130$ bases (without SL) and approximately 400 bases for 3'UTR. These estimates are supported by transcript sequences that were retrieved from the Sequence Read Archive (SRA) section of National Center for Biotechnology Information (transcriptome reads). We found short transcript sequences that partially map to the extremities of both UTR. The SRA reads accessions are 5' UTR - SRR799814.5318050, SRR1118380.5167076 and 3' UTR - SRR1118380.6178627, SRR1118380.7236671, SRR1118380.5954204, SRR1118380.3343870.

The UTR sequences - Approximately 160 bases for 5'UTR (including $39 \mathrm{nt}$ of SL) and 400 bases for 3'UTR were submitted to ProbablePair algorithm in the RNAstructure Web Server (rna.urmc.rochester.edu/RNAstructureWeb/) (Reuter \& Mathews 2010). The predicted bp were selected at $97 \%$ threshold for 5'UTR and 95\% for 3'UTR. These were the highest values upon which the bp arise in all sequences in the analysed strains. To plot the predicted bp arc diagrams, we used the program R-chie (e-rna.org/r-chie/) (Lai et al. 2012). In order to evaluate the presence of conserved or potentially active motifs in the 3'UTR, the segments containing predicted bp were used to both searching an UTR database and scanning 
by RNA motifs finder programs. The databases and motifs finder programs are described below. Searching and scanning were carried out under the parameters suggested by the software developer: (i) UTR database (UTRdb) (utrdb.ba.itb.cnr.it/), (ii) CMfinder 2.0 web server (wingless.cs.washington.edu/htbin-post/unrestricted/CMfinderWeb/CMfinderInput.pl) (Yao et al. 2006), (iii) MEME software (meme.sdsc.edu) (Bailey et al. 2009).

Statistics - The statistical analysis regarding differences in transcript abundance of TCABCG1 gene between BZ-susceptible and BZ-resistant strains was performed with the Student's $t$ test. The test was applied with a confidence interval (CI) of $95 \%(\mathrm{p}=0.05)$ to accept the null hypothesis $\left(\mathrm{H}_{0}\right)$ (there is no significant difference in the transcript levels between the 2 groups of strains). Oneway ANOVA followed by Holm-Sidak multiple comparisons method was employed to analyse the differences of BZ sensitivity between the groups of transfected parasites vs. a control group. Overall significance level $=0.05$. All the data were analysed with SigmaPlot v.11.0.

\section{RESULTS}

Variability of BZ sensitivity among T. cruzi strains - The sensitivity to BZ was determined in epimastigote forms of eight strains belonging to four DTUs (TcI, TcII, $\mathrm{TcV}$ and TcVI) (Table I). These DTUs are predominant in Chagas disease patients in different regions of Latin America (Miles et al. 2009, Zingales et al. 2012). We observed up to five-fold variation of the $\mathrm{IC}_{50}$ values to $\mathrm{BZ}$ among the strains (115 strain, $7.6 \pm 1.6 \mu \mathrm{M}$; YuYu strain, $40.5 \pm 1.8 \mu \mathrm{M})$. The rationale for considering a strain susceptible or resistant to BZ has been discussed elsewhere (Moreno et al. 2010) and was based on the agreement between the drug activity in vitro and in vivo (Filardi \& Brener 1987).

One ABC transporter is over-expressed in BZ-resistant strains - The differential gene expression between BZ-resistant and BZ-susceptible strains was initially investigated by the hybridisation of CL Brener DNA microarray slides with epimastigote cDNAs of the VL10 $\left(\mathrm{IC}_{50} 30.4 \pm 2.9 \mu \mathrm{M}\right)$ and the $115\left(\mathrm{IC}_{50} 7.6 \pm 1.6 \mu \mathrm{M}\right)$ strains. These strains were chosen since both were isolated from patients in the chronic phase of Chagas disease in the state of Minas Gerais, Brazil. Analysis of the hybridisation data indicated that 578 probes were up-regulated in the VL10 strain and 676 probes up-regulated in the 115 strain. The list describing these probes will be published elsewhere (M Moreno et al., unpublished observations). Among the probes up-regulated in VL10 there were two oligonucleotides, QTC00005_L_15 and QTC00014_D_3, showing cDNA hybridisation ratios VL10/115 $1 . \overline{8}$ and 3.6, respectively. These probes (ftp.jcvi.org/pub/data/PFGRC/MAIN/microarray/annotation/T_cruzi/version1/T_ cruzi_1_CLBrener.txt) represent different regions of the same putative $\mathrm{ABC}$ transporter gene of CL Brener nonEsmeraldo-like haplotype (GenBank XM_813521.1). It should be pointed out that oligonucleotides representing the Esmeraldo-like haplotype of the same gene (GenBank XM_801573.1) were not spotted on the microarray slide. As described by El-Sayed et al. (2005), CL Brener is a hy- brid strain and the two haplotypes, corresponding to the two ancestral genomes, were named Esmeraldo-like and non-Esmeraldo-like. Here, the two haplotypes will be abbreviated as Esmo and NEsmo, respectively.

The identified ABC transporter gene (1,998 bp) is a single copy gene located on chromosome 37 of CL Brener (Weatherly et al. 2009). The sequence of the transporter protein (665 residues), encoded by the NEsmo haplotype (XP_818614), and the secondary structure, predicted with the SOSUI system (harrier.nagahama-ibio.ac.jp/sosui/), are shown in Fig. 1. According to this scheme (Fig. 1B), the NBD would be comprised between residues 1- 400. The ATP-binding site, spanning from residues 74-296, contains the Walker A, ABC signature and Walker B motifs. The six presumed TMD $\alpha$-helices are indicated in the Fig. 1. Based on these features, we concluded that the transporter belongs to the half-size ABCG family (NBD-TMD structure arrangement). Being the first ABCG transporter to be characterised in $T$. cruzi it was named TcABCG1.

To validate the differential gene expression, the relative transcript abundance of TCABCG1 was determined by real time RT-PCR in three BZ-susceptible and five BZresistant strains (Fig. 2). CL Brener was used as the reference organism (relative abundance 1). To verify if the differences in transcript abundance of TcABCG1 between the two groups of strains (BZ-susceptible and BZ-resistant) were statistically significant, the data were submitted to the Student's $t$ test. The test was applied with a $95 \% \mathrm{CI}(\mathrm{p}=$ 0.05 ) to accept the $\mathrm{H}_{0}$. The analysis indicated that the separation between the two groups was statistically significant $(\mathrm{t}=-2.935)$, allowing us to conclude that the BZ-resistant strains had higher levels of TCABCG1 transcripts.

Variation of TcABCG1 protein sequence in T. cruzi strains - Next we verified whether, besides differences in the level of expression of TCABCG1 gene among the strains, there would be also sequence variations. The sequence of the TcABCG1 transporter of the Silvio strain is available (GenBank EKG04318.1). Then we sequenced the TCABCG1 gene of the YuYu strain (GenBank KM520157), which is more resistant to BZ than Silvio and also belongs to DTU TcI. Sequence alignment of the two genes showed six single nucleotide polymorphisms (SNPs) (Supplementary Table II), but the ABCG proteins were identical (Table II).

Alignment of the predicted TcI proteins with the protein encoded by the NEsmo haplotype indicated 11 amino acid residue changes (Table II), whose localisation is indicated in Fig. 1B. Three variations were verified before the putative ATP-binding site. In this site, Ser80 of NEsmo was substituted by Ala in TcI strains. As expected, no variation occurred in the Walker A and B motifs or the $\mathrm{ABC}$ signature. Three amino acid changes were observed after the ATP binding site: Gly375, Arg376 and Met394 of NEsmo were replaced, respectively, by Ser, Met and Ile in Silvio and YuYu strains. The differences in the charge of the lateral chains of the amino acid residues may introduce some structural modifications. In the TMD, which seems to confer specificity to the 
A

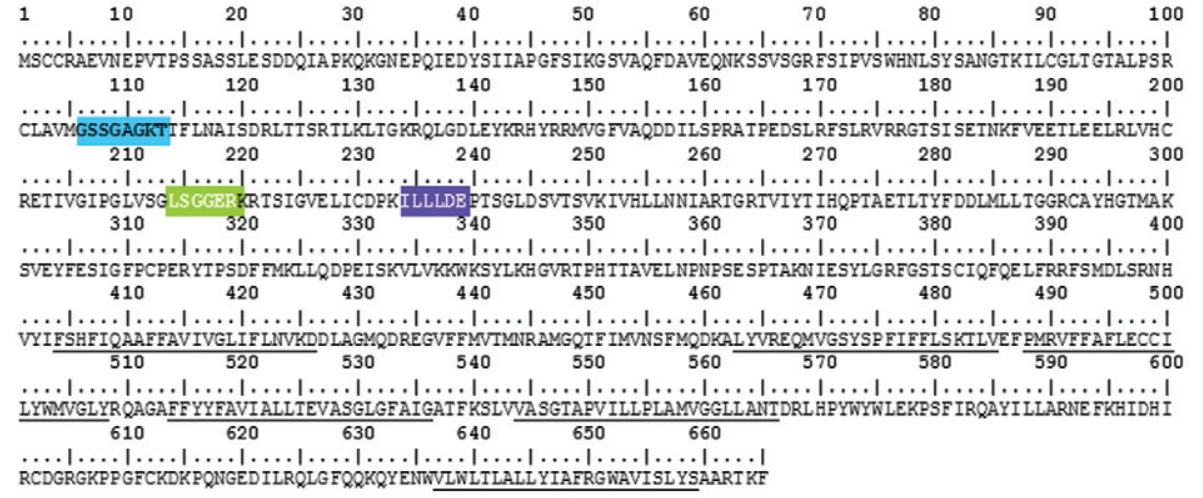

B RCDGRGKPPGFCKDKPQNGED ILRQLG FQQKQYENWVLWLTLALL YIAFRGWAVISL YSAART KF

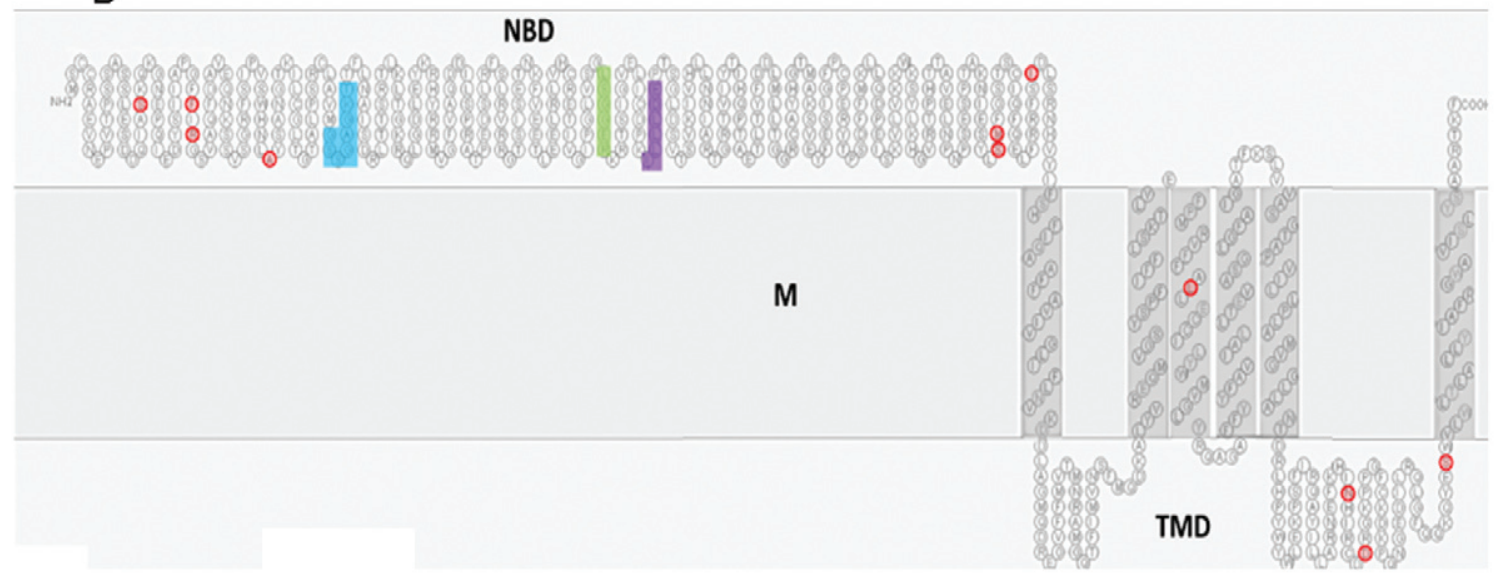

Fig. 1: protein sequence of TcABCG1 transporter (665 residues) encoded by CL Brener NEsmo haplotype. Rectangle blue, Walker A motif, green, $\mathrm{ABC}$ signature and purple, Walker B motif. The putative localisation of the six $\alpha$-helices is underlined; B: secondary structure of TcABCG1 predicted with the SOSUI system (harrier.nagahama-i-bio.ac.jp/sosui/). The localisation of nucleotide binding domain (NBD), membrane (M) and transmembrane domain (TMD) is indicated. Within the NBD, Walker A motif, ABC signature and Walker B motif are indicated as above. In the TMD, the six $\alpha$-helices are shown in grey. Red circles denote the amino acid residues that differ between TcI strains and NEsmo (Table II).

molecule to be translocated (Velamakanni et al. 2007), no amino acid changes occurred in five of the six putative $\alpha$-helices (Fig. 1B). In the third $\alpha$-helix, Phe 495 was replaced by Leu. This is a conservative change of hydrophobic amino acids and unlikely to have a major effect on function. Three amino acid substitutions were observed in the region between the fifth-sixth $\alpha$-helices: Asp598, Gly604 and Asn635 of NEsmo, substituted, respectively, by Asn, Asp and Ser in TcI strains.

Participation of TcABCG1 transporter in BZ natural resistance - To investigate the possible influence of TcABCG1 transporter structure in the sensitivity to $\mathrm{BZ}$, we transfected the genes of the two TcI BZ-resistant strains (Silvio and $\mathrm{YuYu}$ ) and the NEsmo haplotype in BZ-susceptible CL Brener epimastigotes. The genes were cloned in pROCK.Neo plasmid (Fig. 3A), which can be integrated in any of T. cruzi multiple $\beta$-tubulin loci (DaRocha et al. 2004). As controls the parasites were also transfected with the empty vector or PBS. The transfected parasites were submitted to drug selection with $200 \mu \mathrm{g} / \mathrm{mL}$ G418 for eight weeks, when all the parasites electroporated with PBS died.

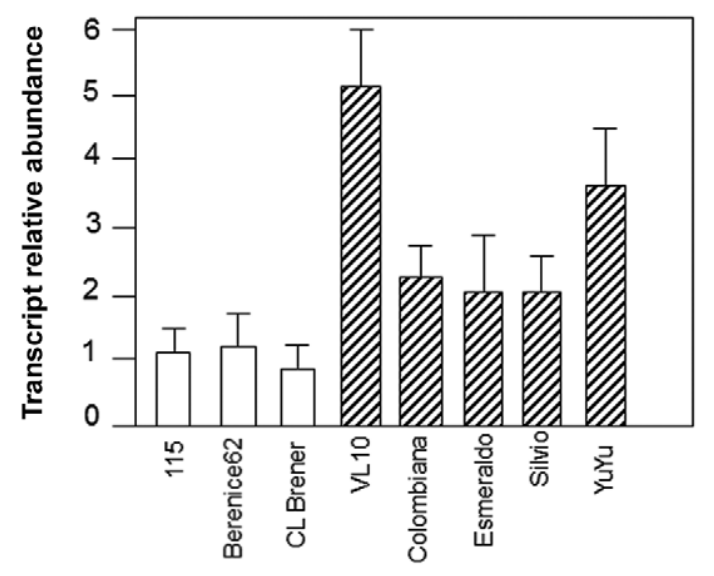

Fig. 2: relative transcript abundance of $T c A B C G 1$ transporter in epimastigote forms of benznidazole (BZ)-susceptible (white rectangles) and BZ-resistant (hatched rectangles) strains. The data represent the mean and standard deviation of two biological replicates with triplicates in each assay. CL Brener (NEsmo haplotype) was used as reference (relative abundance 1). 
TABLE II

Variation of amino acid residues in the TcABCG1 transporter ${ }^{a}$

\begin{tabular}{|c|c|c|c|c|c|c|c|c|c|c|c|c|c|c|c|c|c|c|}
\hline Strains & GenBank & 28 & 48 & 50 & 80 & 124 & 375 & 376 & 382 & 394 & 462 & 495 & 507 & 598 & 604 & 611 & 635 & 637 \\
\hline Silvio & EKG0431 & $\mathrm{S}$ & $\mathrm{F}$ & $\mathrm{R}$ & $\mathrm{A}$ & $\mathrm{T}$ & S & M & $\mathrm{C}$ & I & A & $\mathrm{L}$ & $\mathrm{L}$ & $\mathrm{N}$ & $\mathrm{D}$ & $\mathrm{F}$ & $\mathrm{S}$ & V \\
\hline $\mathrm{YuYu}$ & KM52015 & . & . & . & . & . & . & . & . & . & . & . & . & . & . & . & . & . \\
\hline CLB.NEsmo & XP_818614.1 & $\mathrm{P}$ & $\mathrm{S}$ & $\mathrm{K}$ & $\mathrm{S}$ & . & G & $\mathrm{R}$ & . & M & . & $\mathrm{F}$ & . & $\mathrm{D}$ & G & . & $\mathrm{N}$ & . \\
\hline CLB.Esmo & XP_806666.1 & . & $\mathrm{S}$ & $\mathrm{K}$ & . & $\mathrm{K}$ & . & $\mathrm{R}$ & G & . & $\mathrm{P}$ & F & $\mathrm{F}$ & $\mathrm{D}$ & G & $\mathrm{Y}$ & . & I \\
\hline
\end{tabular}

$a$ : the amino acid position refers to the protein sequence of Silvio X10 cll strain; $b$ : position of nucleotide binding domain (NBD) and transmembrane domain (TMD) inferred with the SOSUI system (Fig. 1B). The dots represent the same amino acid residues as Silvio.

The integration of the exogenous $T c A B C G 1$ genes in CL Brener genome was investigated by Southern blot. Total DNA of CL Brener wild type (WT), Silvio WT and transfectants was digested with BamHI and separated in an agarose gel (Fig. 3B). The blot was hybridised to a probe represented by the mixture of a 616-bp DNA fragment of the TCABCG1 gene of CL Brener and Silvio strains (Fig. $3 \mathrm{C}$ ). This procedure was necessary since there are differences in the nucleotide sequence of this region between the two strains. The probe identified a high molecular mass band $(\sim 21 \mathrm{~kb})$ in the WT strains and CL Brener transfected with the empty plasmid. In $T C A B$ $C G 1$ transgenes a doublet was verified around $21 \mathrm{~kb}$ : one corresponding to the CL Brener endogenous gene and the other, most probably, to the introduced gene. Two additional bands were also seen, one of molecular mass higher than $21 \mathrm{~kb}$ and another of $\sim 7 \mathrm{~kb}$.

Hybridisation of BamHI-digested DNA (Fig. 3D) with a probe derived from $\mathrm{pROCK}$.Neo gene that confers $\mathrm{NeoR}$, confirmed the integration of the vector in the parasite genomes (Fig. 3E). In the transfectants bearing the transporter gene, three bands were identified (Fig. 3E) of the same size as the bands recognised by the TcABCG1 probe in the transgenes (Fig. 3C). As expected, the neomycin probe did not hybridise with CL Brener WT DNA. A weak band of molecular mass $\sim 21 \mathrm{~kb}$ was observed in CL Brener transfected with the empty pROCK vector.

T. cruzi genes encoding $\alpha$ and $\beta$-tubulin are arranged in a cluster with an alternating $\alpha$ and $\beta$-array with a basic repeat unit length of $4.3 \mathrm{~Kb}$ (Maingon et al. 1988). In addition, other pairs of alternating $\alpha$ and $\beta$-tubulin sequences appear to be physically separated from the basic group (Maingon et al. 1988). Each $\beta$-tubulin gene has one BamHI restriction site. The three bands observed in the transgenic parasites hybridising with $T C A B C G 1$ and neomycin probes are the result of the insertion of pROCK.Neo in one of the many $\beta$-tubulin copies. As a whole, the Southern blot data confirmed that the exogenous $T c A B C G 1$ genes were integrated in CL Brener genome, most probably in the same locus.

BZ susceptibility in the transfected parasites and CL Brener WT was determined. Data in Fig. 4A refer to the $\mathrm{IC}_{50}$ values (mean \pm standard deviation). In relation to $\mathrm{CL}$ Brener WT, we verified $47 \%$ and $41 \%$ increase of the $\mathrm{IC}_{50}$ values in the cultures transfected with the TCABCGl gene of

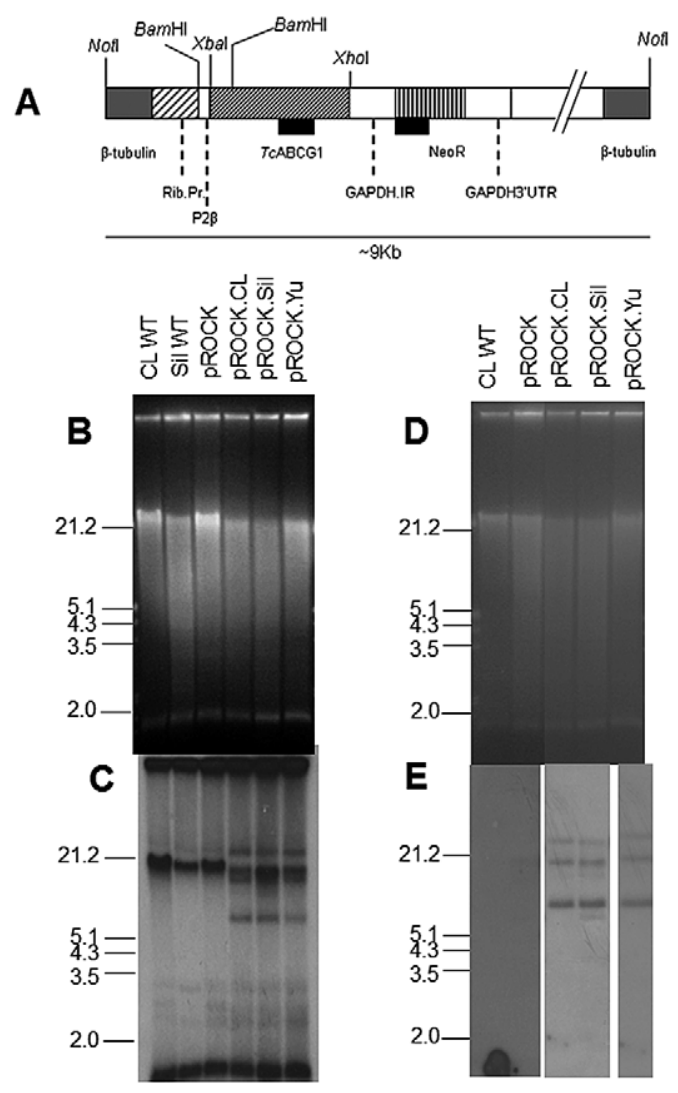

Fig. 3A: schematic representation of the pROCK.Neo TcABCG1 vector construct. The $\beta$-tubulin locus is followed by the Trypanosoma cruzi ribosomal promoter and the $\mathrm{P} 2 \beta$ 5' intergenic region for spliced leader addition. The open reading frame of $T c A B C G 1$ gene was cloned between the $X b a \mathrm{I}$ and $X h o \mathrm{I}$ unique restriction sites, followed by the 3 ' untranslated region plus intergenic region derived from the glyceraldehyde 3-phosphate dehydrogenase I gene. The localisation of the neomycin resistance (NeoR) gene used as a drug-selectable marker is indicated. Black rectangles denote the localisations of the 616-base pair (bp) probe of the TcABCG1 gene and the 435-bp probe of the $\mathrm{NeoR}$ gene, both used in the Southern blot. The unique restriction site for the enzyme NotI was used for vector linearisation; B-E: Southern blot of total DNA digested with BamHI. Ethidium bromide stained gels $(\mathrm{B}, \mathrm{D})$ and hybridisations with ${ }^{32} \mathrm{P}$-labelled probes 616-bp DNA fragment of the TCABCGI gene (C) and NeoR pROCK gene (E); E: the membrane strips corresponding to CL Brener wild type (WT), empty pROCK and pROCK.Yu were exposed for longer times since the hybridisation signal. Molecular mass markers are indicated in $\mathrm{kb}$. 
A

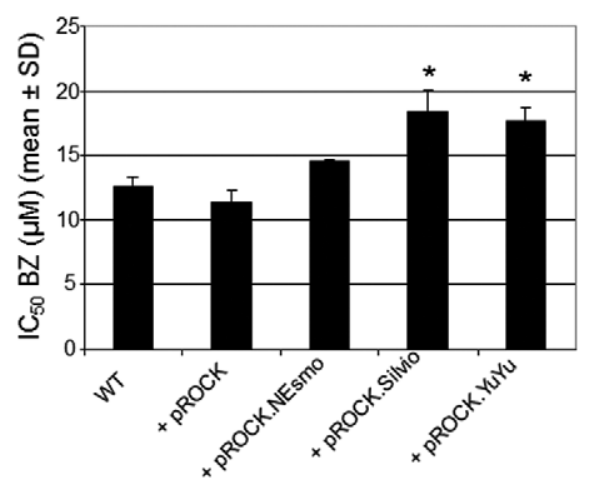

B

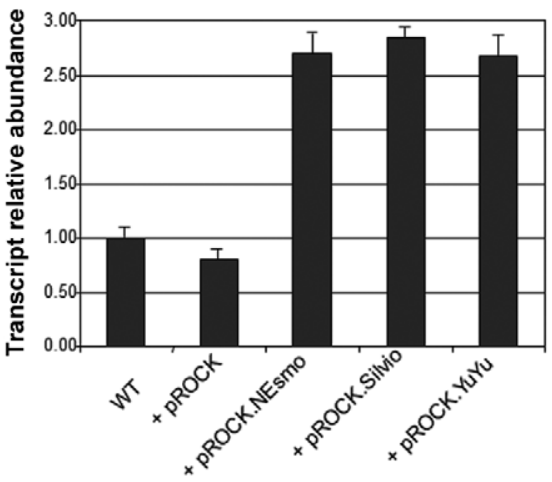

Fig. 4: susceptibility to benznidazole (BZ) (A) and transcript relative abundance (B) in CL Brener epimastigotes transfected with the TcABCG1 gene of CL Brener NEsmo, Silvio and YuYu strains cloned in pROCK.Neo vector. The parameters were also obtained for CL Brener wild type (WT) and parasites transfected with the empty vector. Mean and standard deviation (SD) are indicated and were obtained from at least three independent assays (biological replicates) with three replicates in each assay. Asterisks mean significant values compared to CL Brener WT. $\mathrm{IC}_{50}: 50 \%$ inhibitory concentration.

TABLE III

One-way ANOVA followed by Holm-Sidak multiple comparisons method was used to analyse the differences in the mean values of benznidazole sensitivity between the transfected cultures and CL Brener wild type (WT) (Fig. 3)

\begin{tabular}{|c|c|c|c|c|c|}
\hline Comparison & $\begin{array}{l}\text { Difference } \\
\text { of means }\end{array}$ & $\mathrm{t}$ & $\begin{array}{c}\text { Unadjusted } \\
\text { p }\end{array}$ & $\begin{array}{c}\text { Critical } \\
\text { level }\end{array}$ & Significant? $^{a}$ \\
\hline CL WT vs. empty pROCK & 1.221 & 1.644 & 0.121 & 0.050 & No \\
\hline CL WT vs. pROCK.CL & 2.007 & 2.482 & 0.025 & 0.025 & No \\
\hline CL WT vs. pROCK.Silvio & 5.917 & 7.965 & $<0.001$ & 0.013 & Yes \\
\hline CL WT vs. pROCK.YuYu & 5.167 & 6.955 & $<0.001$ & 0.017 & Yes \\
\hline
\end{tabular}

$a$ : there is a statistically significant difference when $\mathrm{p} \leq 0.001$; overall significance level: 0.05 .

Silvio and YuYu strains, respectively. In the culture transfected with CL Brener gene the $\mathrm{IC}_{50}$ increase was $16 \%$.

The one-way ANOVA followed by Holm-Sidak multiple comparisons method were employed to analyse the differences in the mean values of $\mathrm{BZ}$ sensitivity $\left(\mathrm{IC}_{50}\right.$ values) between the transfected cultures and CL Brener WT (control group) (Table III). We concluded that the differences in the mean $\mathrm{IC}_{50}$ values of the parasites transfected with the Silvio and $\mathrm{YuYu}$ genes are greater than would be expected by chance $(\mathrm{p} \leq 0.001)$. On the other hand, the increase of the resistance to the drug in parasites transfected with the homologous NEsmo haplotype or the empty vector had no statistical significance $(\mathrm{p}=$ 0.025 and 0.121 , respectively).

Relative abundance of TcABCG1 transcripts and transporter protein in CL Brener transfected parasites - The relative abundance of the TCABCG1 transcripts in the transfected parasites was determined by real time RT-PCR (Fig. 4B). An 2.7-fold increase was verified in the parasites transfected with pROCK recombinant vectors. No significant statistical difference was verified regarding the strain source of the TcABCG1 gene: $\mathrm{CL}$ Brener, Silvio or YuYu (ANOVA test $\mathrm{p}<0.05$ ).

To estimate the levels of TcABCG1 transporter protein, we obtained an antiserum to a protein region comprised between the amino acid residues 77 and 320. This region $(\sim 26 \mathrm{kDa})$ contains the Walker $\mathrm{A}$ and $\mathrm{B}$ motifs and the ABC signature. The immunoblot of total protein of CL Brener WT and transfected parasites incubated with the anti-TcABCG1 serum showed the recognition of an $\sim 76 \mathrm{kDa}$ band, corresponding to the TcABCG1 protein (Fig. 5A). To correct for differences in signal intensities due to variations in the amount of protein loaded in each lane, the blot was incubated with an antiserum to T. cruzi gGAPDH. Densitometric quantifications of the signals allowed us to conclude 2-2.6-fold increase of the TcABCG1 protein in the cultures electroporated with the TcI gene of Silvio and YuYu strains, respectively (Fig. 5A). In CL Brener transfected with the NEsmo haplotype an increase of only 1.1 fold was observed.

Transporter abundance in natural BZ-resistant and susceptible strains - The anti-TcABCG1 serum was 

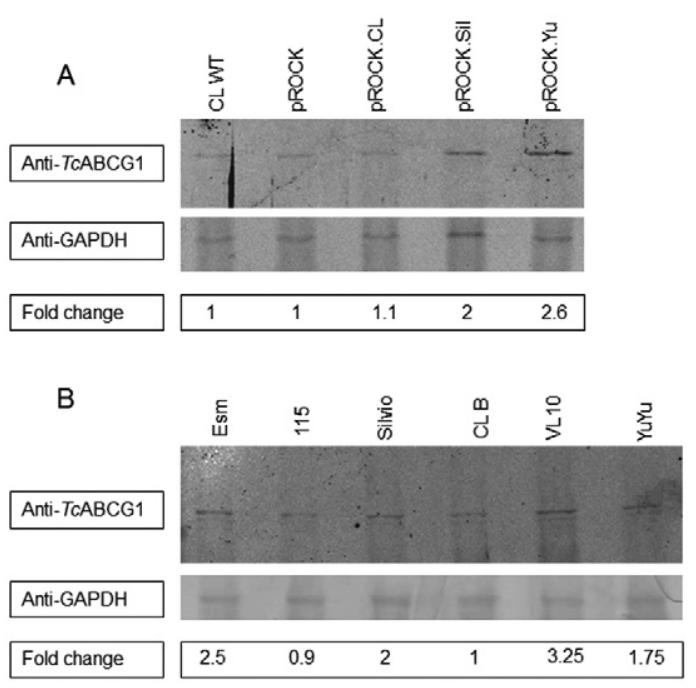

Fig. 5: western blot with total protein of T. cruzi epimastigotes. A: CL Brener wild type (WT) and CL Brener pROCK-transfected parasites; B: WT strains. In each panel, membranes were incubated with an antiserum against a region of the TCABCG1 transporter. Subsequently, the blot was incubated with an antiserum to T. cruzi gGAPDH to assess protein levels. Densitometric quantification of the signals obtained with the two antibodies allowed us to estimate the increased abundance of $T c$ ABCG1 transporter in relation to CL Brener WT; GAPDH: glyceraldehyde 3-phosphate dehydrogenase.

employed to determine differences in the protein levels among WT strains (Fig. 5B), which showed variations in TcABCG1 transcript levels (Fig. 2). Following incubation with the anti-gGAPDH serum, densitometric quantifications of the signals indicated 2-3-fold higher abundance of the TcABCG1 transporter in strains with increased resistance to $\mathrm{BZ}$, as compared to $\mathrm{CL}$ Brener and 115 sensitive strains (Fig. 5B). We observed a reasonable correlation between the relative abundance of TCABCG1 transcripts and protein levels (Fig. 2).

Analysis of 5'UTR and 3'UTR of TcABCG1 genes - In kinetoplastids, regulation of gene expression is exerted at the posttranscriptional level (Clayton \& Shapira 2007) and in trypanosomatids, specific motifs and ordered secondary structures in 5' and 3' UTRs may control mRNA half-life and translation efficiency (Brandão 2006, Clayton \& Shapira 2007, Siegel et al. 2011, Clayton 2014).

Here we analysed in silico characteristics of the UTRs of TcABCG1 genes of Silvio, Esmeraldo and CL Brener (Esmo and NEsmo), since these strains differ in BZ sensitivity (Table I), transcript abundance (Fig. 2) and protein levels (Fig. 5B).

The genomic locus of TcABCG1 displays the same configuration in the three strains. It is flanked at the 5 , upstream by the 40S ribosomal protein S8 (RPS8) and at 3 ' downstream by a hypothetical protein. The intergenic segment separating the 40S RPS8 and the TcABCG1 transporter in CL Brener NEsmo and Esmo haplotypes, Silvio and Esmeraldo strains is 526, 524, 520 and 519 bases, respectively. Intergenic mutations change both the length and nucleotide composition of the 5' UTR, which we estimated as having length of 116, 127, 121 and 123 bases, respectively, for NEsmo and Esmo haplotypes, Silvio and Esmeraldo strains. 5'UTRs were investigated with respect to the potential of generating high probability bp (through the ProbablePair algorithm). Firstly we inspected the base pairing at the highest probability threshold in each sequence. The ProbablePair algorithm allows a prediction that whatever the real conformation in that segment of the mRNA, it is almost certain that the predicted paired bases are present in the actual secondary structure. The four 5'UTR sequences analysed here exhibit paired bases at the threshold limit of $70 \%$, that is, in the thermodynamical ensemble of secondary structures, those paired bases were present in $70 \%$ of all configurations (Fig. 6A). The low compositional variation in the 5'UTR of each strain determines the trend to be more or less structured. Apparently, the naturally BZ resistant strains (Silvio and Esmeraldo) exhibit more paired bases than both haplotypes of CL Brener BZ sensitive strain (Fig. 6A, Table IV).

The 3'UTR sequences were initially used as query to search an UTR database for similarity to conserved and functional elements from other eukaryotes. This search however, returned no elements except for the detection of two small ORFs (data not shown). Thus, the ABCG transporter 3'UTR does not possess similarity to any evolutionary conserved UTR element described so far. We next submitted the 3'UTR sequences to ProbablePair algorithm for base pairing prediction at $95 \%$ probability threshold. The predicted bp at this elevated threshold indicates that they are highly structured sequences, which is evidence that stem loop elements are almost certainly present in any secondary structure these 3'UTR might assume. A description of helix position in these sequences is available at Supplementary Table III. We then scanned the 3'UTR sequences with two RNA motif finder software (CM finder and MEME) that returned a set of three motifs each, covering more than half of 3'UTR sequence. Fig. 6B shows a diagram of detected motifs and the predicted bp for all 3'UTR segments. By this figure we see that the three algorithms produced an overlapping prediction in the segment defined by nucleotide positions 60-100 and only for two sequences (Esmeraldo and Esmo haplotype). Though the algorithm did not detect simultaneously the same element in all four sequences, the overlapping of motifs predicted by two different algorithms is a good hint that the segment spanning these motifs might be at least a hot spot for some functional activity. By this reasoning, the segment between positions 140-220 (Fig. 6B) is the one with high potential for a functional activity, because it contains several overlapping bp and has been assigned two motifs by $\mathrm{CM}$ finder. Analog reasoning is valid for the segment 10-100. Supplementary Table IV describes these predicted minimum common motifs. The structured nature of the 5'UTR, the highly probable stem loops and the potential sequence motifs pointed out by the in silico analysis of TcABCG1 3'UTRs are very suggestive that 


\section{A - 5'UTR}

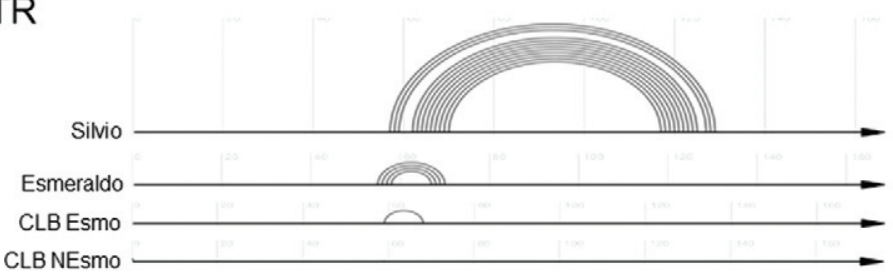

B - 3'UTR
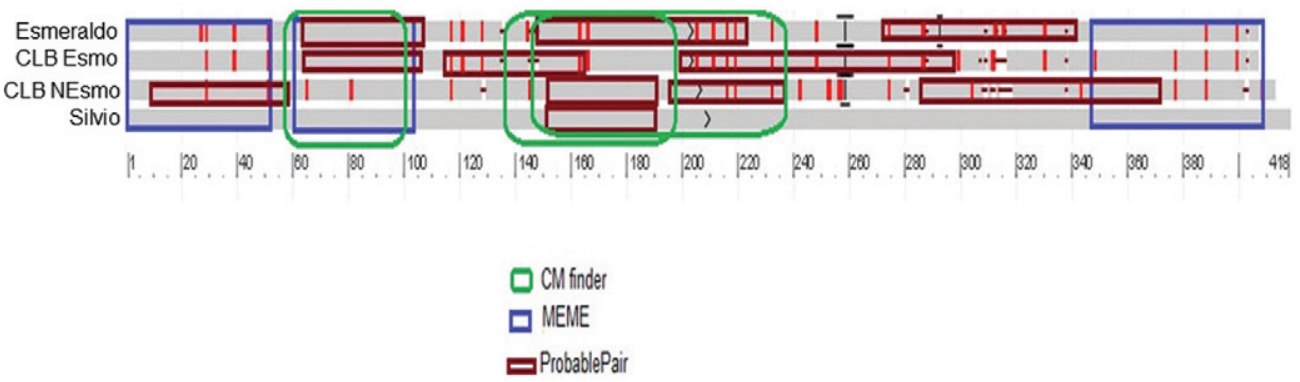

Fig. 6A: arc diagram representation of the predicted base pairs (bp) by the ProbablePair algorithm at $70 \%$ probability threshold in the estimated 5 , untranslated region (UTR) sequences of Trypanosoma cruzi strains. A decreasing in the potential to form secondary structures in these sequences is observed from the benznidazole (BZ)-resistant strains (Silvio and Esmeraldo) to the BZ-sensitive strain (CL Brener Esmo and NEsmo); B: schematic representation of the predicted bp and potential motifs/elements detected by the algorithms ProbablePair, CM finder and MEME in the 3'UTR sequences; grey bars in the background: 400 bases of each 3'UTR; red vertical bars: nucleotide substitution sites; horizontal rectangles: predicted bp by ProbablePair at 95\% threshold probability; large blue and green rectangles: position of elements identified respectively by MEME and CM finder.

\section{TABLE IV}

High probability base pairs (bp) in the 5' untranslated region (UTR) of TcABCG1 transporter ${ }^{a}$

\begin{tabular}{lcccc}
\hline Strain & bp & $\begin{array}{c}\text { Helix position in 5'UTR } \\
\left(160 \mathrm{nt}^{b}\right)\end{array}$ & $\begin{array}{c}\text { Stem } \\
\text { loops }\end{array}$ & $\begin{array}{c}\text { BZ } \\
\text { phenotype }\end{array}$ \\
\hline Silvio & 12 & $57-70 ; 117-129$ & 2 & $\mathrm{R}$ \\
Esmeraldo & 4 & $55-70$ & 1 & $\mathrm{R}$ \\
CL Brener Esmo & 1 & $59-68$ & 1 & $\mathrm{~S}$ \\
CL Brener NEsmo & 0 & - & 0 & $\mathrm{~S}$
\end{tabular}

$a$ : paired bases appearing at the maximum threshold of $70 \%$ (ProbablePair algorithm); $b$ : including spliced leader; BZ: benznidazole; R: resistant; S: susceptible.

the transcript abundance and resistance/sensitivity to BZ might be directly influenced by the variable composition of UTRs in different T. cruzi strains.

\section{DISCUSSION}

In this study we provide evidence that the ABCG-like transporter TcABCG1 plays a role in T. cruzi natural resistance to BZ. Initial indications for this were obtained from the observation that in naturally drug-resistant strains there was an increased level of TcABCG1 gene transcripts.

We have generated CL Brener cell lines in which $T C A B$ $C G 1$ genes from two TcI BZ-resistant strains were independently transfected in the BZ-susceptible CL Brener strain. We verified that the transfected parasites acquired $\sim 45 \%$ increase of BZ-resistance. Interestingly, no statistically significant increment in drug resistance was verified in CL Brener transfected with the self NEsmo haplotype. Comparison of the nucleotide sequence of $T c A B C G 1$ gene of the TcI strains with the sequence of NEsmo haplotype showed several SNPs that produced 11 amino acid changes.

TcABCG1 transporter exhibits 29\% similarity with the ABCG2 human transporter, also known as breast cancer resistance protein (Doyle et al. 1998). This transporter plays a role in multidrug resistance to chemotherapeutic agents. Amino acid changes have been identified in ABCG2, some of which affect the expression level, transporter function and/or cellular localisation (Kondo et al. 2004, Yanase et al. 2006). 
We have shown that irrespective of the origin of the introduced gene, in the transfected parasites there was $\sim 2.7$-fold increased abundance of TCABCG1 transcripts, as compared to CL Brener WT. The conserved increment of transcript abundance most likely results from the cloning of TcABCG1 genes in pROCK vector, in which the ORFs are flanked by the same 5' and 3' UTR sequences (DaRocha et al. 2004).

The finding that there was 2-2.6-fold increased levels of the transporter protein only in parasites transfected with TcI genes, although in agreement with the observed enhancement of drug resistance, is intriguing. Two major hypotheses could be raised to justify the results. The first one implies that characteristics of the sequence of the coding region may be influencing the translation or the steady state levels of TcI proteins. The second one argues about the expression of the protein encoded by the NEsmo haplotype. Comparison of the gene and protein sequence of Esmo and NEsmo haplotypes showed several SNPs (Supplementary Table II) and 11 amino acid changes (Table II). In addition, structural differences were verified in the 5' and 3' UTRs of the two genes (Fig. 6, Table IV). It is known that G-family transporters undergo dimerisation to perform their function (Velamakanni et al. 2007). On the other hand, it is not known if both or only one haplotype is expressed in CL Brener or if the functional TcABCG1 transporter may be formed by the dimerisation of dissimilar subunits. In the future, it will be of interest to verify if the abundance of the transporter protein increases when CL Brener is transfected with the Esmo gene.

Although CL Brener transfected with TcI genes showed $\sim 45 \%$ increased resistance to BZ, the parasites did not attain the resistance level observed in TcI WT strains, which is 2-3-fold higher than CL Brener (Table I). This result may be due to the impaired expression of TcI genes in a TcVI strain. In this case, the dimerisation of the transporter and/ or posttranslation modifications could be defective and/or additional DTU-specific elements could be required to act in conjunction with the ABCG transporter.

Our studies with WT strains suggest that the abundance of TcABCG1 transporter is a major factor for the natural resistance to BZ. In fact, we observed a reasonable correlation between the relative abundance of TCABCG1 transcripts and protein levels.

We investigated by computational tools the characteristics of UTRs of TcABCG1 genes of CL Brener, Silvio and Esmeraldo strains that could justify the differential gene expression in the WT strains. We estimated that 5'UTR sequences ( $160 \mathrm{nt})$ of the four genes have low compositional variation and that the sequences of the naturally BZ resistant strains Silvio and Esmeraldo exhibited more paired bases than both haplotypes of CL Brener. Differences in composition and length were verified in the 3'UTR sequences ( 400 nt), which could generate segments with different structural properties. For example, the resistant strain Silvio possesses a slightly longer 3'UTR and exhibits several SNPs in comparison to other strains. Structural differences were also verified between the Esmo and NEsmo haplotypes. In 3'UTRs we detected several motifs, which are overlapping segments with high probability of forming secondary structures (stem loops). This can be viewed as road map to test the hypothesis that natural acquisition of resistance to BZ might be dependent on mutational events occurring in noncoding segments like UTRs. As demonstrated by the variability in predicted bp for the sequences analysed here, a few mutations can alter the positions where a robust base pairing can appear or not in a defined segment. In the future, a more accurate analysis of the UTRs will be performed from the direct sequencing of $T c A B C G 1$ transcripts.

For sure, even if the computational tools indicate the presence of either conserved sequence elements or high probability secondary structures in both UTRs, we cannot rule out the alternative hypothesis that the differential functional activities attributed to UTR (5' and 3') may also be the result of their interaction with trans elements, like proteins or other nucleic acids. In this direction, controlled experiments should be designed to characterise these elements.

Very few ABC genes have been characterised in $T$. cruzi, while almost 30 genes are recognised in the genome of this parasite (Leprohon et al. 2006, Sauvage et al. 2009). The first ABC genes identified in T. cruzi were termed tcpgpl and tcpgp2 (Dallagiovanna et al. 1996, Torres et al. 1999). Because the genes belong to the ABCC family, they were renamed TCABCC6 and TCAB$C C 2$ (Sauvage et al. 2009). No differential expression or DNA polymorphisms of these genes between susceptible and BZ/NF-resistant T. cruzi strains were verified (Murta et al. 2001). One ABCA like-transporter, termed TcAB$\mathrm{CA} 3$, seems to be involved in vesicular trafficking and is differentially expressed throughout the parasite life cycle (Torres et al. 2004). By using specific inhibitors of ABC transporters, the involvement of a P-glycoprotein in the transport of haeme through the plasma membrane of $T$. cruzi has been suggested (Lara et al. 2007).

To our knowledge, TcABCG1 is the first transporter of the $\mathrm{G}$ family to be characterised in T. cruzi. ABCG transporters are represented in Leishmania species. ABCG4 and ABCG6 transporters are involved in phospholipid trafficking and over-expression promoted significant resistance to miltefosine in Leishmania infantum (Castanys-Muñoz et al. 2007, 2008), while ABCG6 is involved in camptothecin resistance in Leishmania donovani (BoseDasgupta et al. 2008).

Homologous sequences of Leishmania ABCG4 and ABCG6 genes exist in Trypanosoma brucei and T. cruzi genomes (Leprohon et al. 2006), but their function has not been established in these organisms. The TCABCG1 transporter here characterised has greater similarity with Leishmania ABCG2 transporter, implicated in phosphatidylserine translocation (Campos-Salinas et al. 2013).

Taken together, the data here presented support the proposition that TcABCG1 is involved in $\mathrm{BZ}$ resistance. Since members of ABCG family have been associated with cellular lipid transport, it is of interest to investigate the physiological role of TcABCG1 in T. cruzi. Work is in progress to assess the role of this transporter in crossresistance to other nitro drugs. This information will be valuable in the decision of Chagas disease treatment with available drugs and drugs to be discovered in the future. 


\section{ACKNOWLEDGEMENTS}

To the Pathogen Functional Genomics Resource Center, for the donation of T. cruzi microarray slides, and to Dr Ariel Silber, for the generous gift of anti-GAPDH serum.

\section{REFERENCES}

Bailey TL, Bodén M, Buske FA, Frith M, Grant CE, Clementi L, Ren J, Li WW, Noble WS 2009. MEME SUITE: tools for motif discovery and searching. Nucleic Acids Res 37: W202-W208.

Baptista CS, Vêncio RZN, Abdala S, Valadares MP, Martins CAO, Zingales B 2004. DNA microarrays for comparative genomics and analysis of gene expression in Trypanosoma cruzi. Mol Biochem Parasitol 138: 183-194.

Baptista CS, Vêncio RZN, Abdala SB, Carranza JC, Westenberger SJ, Silva MN, Pereira CAB, Galvão LMC, Gontijo ED, Chiari E, Sturm NR, Zingales B 2006. Differential transcription profiles in Trypanosoma cruzi associated with clinical forms of Chagas disease: maxicircle NADH dehydrogenase subunit 7 gene truncation in asymptomatic patient isolates. Mol Biochem Parasitol 150: 236-248.

BoseDasgupta S, Ganguly A, Roy A, Mukherjee T, Majumder HK 2008. A novel ATP-binding cassette transporter, ABCG6, is involved in chemoresistance of Leishmania. Mol Biochem Parasitol 158: 176-188.

Brandão A 2006. The untranslated regions of genes from Trypanosoma cruzi: perspectives for functional characterization of strains and isolates. Mem Inst Oswaldo Cruz 101: 775-777.

Brandão A, Jiang T 2009. The composition of untranslated regions in Trypanosoma cruzi genes. Parasitol Int 58: 215-219.

Campos PC, Bartholomeu DC, DaRocha WD, Cerqueira GC, Teixeira SMR 2008. Sequences involved in mRNA processing in Trypanosoma cruzi. Int J Parasitol 38: 1383-1389.

Campos-Salinas J, León-Guerrero D, González-Rey E, Delgado M, Castanys S, Pérez-Victoria JM, Gamarro F 2013. LABCG2, a new $\mathrm{ABC}$ transporter implicated in phosphatidylserine exposure is involved in the infectivity and pathogenicity of Leishmania. PLoS Negl Trop Dis 7: e2179.

Castanys-Muñoz E, Alder-Baerens N, Pomorski T, Gamarro F, Castanys S 2007. A novel ATP-binding cassette transporter from Leishmania is involved in transport of phosphatidylcholine analogues and resistance to alkyl-phospholipids. Mol Microbiol 64: 1141-1153.

Castanys-Muñoz E, Pérez-Victoria JM, Gamarro F, Castanys S 2008. Characterization of an ABCG-like transporter from the protozoan parasite Leishmania with a role in drug resistance and transbilayer lipid movement. Antimicrob Agents Chemother 52: 3573-3579.

Clayton C, Shapira M 2007. Post-transcriptional regulation of gene expression in trypanosomes and leishmanias. Mol Biochem Parasitol 156: 93-101.

Clayton CE 2014. Networks of gene expression regulation in Trypanosoma brucei. Mol Biochem Parasitol 195: 96-106.

Dallagiovanna B, Gamarro F, Castanys S 1996. Molecular characterization of a P-glycoprotein-related tcpgp2 gene in Trypanosoma cruzi. Mol Biochem Parasitol 75: 145-157.

DaRocha WD, Silva RA, Bartholomeu DC, Pires SF, Freitas JM, Macedo AM, Vazquez MP, Levin MJ, Teixeira SMR 2004. Expression of exogenous genes in Trypanosoma cruzi: improving vectors and electroporation protocols. Parasitol Res 92: 113-120.

Doyle LA, Yang W, Abruzzo LV, Krogmann T, Gao Y, Rishi AK, Ross DD 1998. A multidrug resistance transporter from human MCF-7 breast cancer cells. Proc Natl Acad Sci USA 95: 15665-15670.
El-Sayed NM, Myler PJ, Bartholomeu DC, Nilsson D, Aggarwal G, Tran AN, Ghedin E, Worthey EA, Delcher AL, Blandin G, Westenberger SJ, Caler E, Cerqueira GC, Branche C, Haas B, Anupama A, Arner E, Aslund L, Attipoe P, Bontempi E, Bringaud F, Burton P, Cadag E, Campbell DA, Carrington M, Crabtree J, Darban H, da Silveira JF, de Jong P, Edwards K, Englund PT, Fazelina G, Feldblyum T, Ferella M, Frasch AC, Gull K, Horn D, Hou L, Huang Y, Kindlund E, Klingbeil M, Kluge S, Koo H, Lacerda D, Levin MJ, Lorenzi H, Louie T, Machado CR, McCulloch R, McKenna A, Mizuno Y, Mottram JC, Nelson S, Ochaya S, Osoegawa K, Pai G, Parsons M, Pentony M, Pettersson U, Pop M, Ramirez JL, Rinta J, Robertson L, Salzberg SL, Sanchez DO, Seyler A, Sharma R, Shetty J, Simpson AJ, Sisk E, Tammi MT, Tarleton R, Teixeira S, Van Aken S, Vogt C, Ward PN, Wickstead B, Wortman J, White O, Fraser CM, Stuart KD, Andersson B 2005. The genome sequence of Trypanosoma cruzi, etiologic agent of Chagas disease. Science 309: 409-415.

Filardi LS, Brener Z 1987. Susceptibility and natural resistance of Trypanosoma cruzi strains to drugs used clinically in Chagas disease. Trans R Soc Trop Med Hyg 81: 755-759.

Gordon D, Abajian C, Green P 1998. Consed: a graphical tool for sequence finishing. Genome Res 8: 195-202.

Higgins CF 1992. ABC transporters: from microorganisms to man. Annu Rev Cell Biol 8: 67-113.

Higgins CF 2007. Multiple molecular mechanisms for multidrug resistance transporters. Nature 446: 749-757.

Igarashi KF, Aoki H, Mamitsuka K, Kuma M, Kanehisa M 2004. The evolutionary repertoires of the eukaryotic-type $\mathrm{ABC}$ transporters in terms of the phylogeny of ATP-binding domains in eukaryotes and prokaryotes. Mol Biol Evol 21: 2149-2160.

Klokouzas A, Shahi S, Hladky SB, Barrand MA, van Veen HW 2003. $\mathrm{ABC}$ transporters and drug resistance in parasitic protozoa. Int $J$ Antimocrob Agents 22: 301-317.

Kondo C, Suzuki H, Itoda M, Ozawa S, Sawada J, Kobayashi D, Ieiri I, Mine K, Ohtsubo K, Sugiyama Y 2004. Functional analysis of SNPs variants of BCRP/ABCG2. Pharm Res 21: 1895-1903.

Lai D, Proctor JR, Zhu JYA, Meyer IM 2012. R-chie: a web server and $\mathrm{R}$ package for visualizing RNA secondary structures. Nucleic Acids Res 40: e95.

Lara FA, Sant'anna C, Lemos D, Laranja GA, Coelho MG, Salles IR, Michel A, Oliveira PL, Cunha-E-Silva N, Salmon D, Paes MC 2007. Heme requirement and intracellular trafficking in Trypanosoma cruzi epimastigotes. Biochem Biophys Res Commun 355: 16-22.

Leprohon P, Legare D, Girard I, Papadopoulou B, Ouellette M 2006. Modulation of Leishmania ABC protein gene expression through life stages and among drug-resistant parasites. Eukaryot Cell 5: 1713-1725.

Maingon R, Gerke R, Rodriguez M, Urbina J, Hoenicka J, Negri S, Aguirre T, Nehlin J, Knapp T, Crampton J 1988. The tubulin genes of Trypanosoma cruzi. Eur J Biochem 171: 285-291.

Miles MA, Llewellyn MS, Lewis MD, Yeo M, Baleela R, Fitzpatrick S, Gaunt MW, Mauricio IL 2009. The molecular epidemiology and phylogeography of Trypanosoma cruzi and parallel research on Leishmania: looking back and to the future. Parasitology 136: 1509-1528.

Moncayo A, Silveira AC 2009. Current epidemiological trends for Chagas disease in Latin America and future challenges in epidemiology, surveillance and health policy. Mem Inst Oswaldo Cruz 104 (Suppl. I): 17-30. 
Moreno M, D'ávila DA, Silva MN, Galvão LMC, Macedo AM, Chiari E, Gontijo ED, Zingales B 2010. Trypanosoma cruzi benznidazole susceptibility in vitro does not predict the therapeutic outcome of human Chagas disease. Mem Inst Oswaldo Cruz 105: 918-924.

Murta SM, dos Santos WG, Anacleto C, Nirdé P, Moreira ES, Romanha AJ 2001. Drug resistance in Trypanosoma cruzi is not associated with amplification or overexpression of P-glycoprotein (PGP) genes. Mol Biochem Parasitol 117: 223-228.

Murta SM, Gazzinelli RT, Brener Z, Romanha AJ 1998. Molecular characterization of susceptible and naturally resistant strains of Trypanosoma cruzi to benznidazole and nifurtimox. Mol Biochem Parasitol 93: 203-214.

Rassi Jr A, Rassi A, de Rezende JM 2012. American trypanosomiasis (Chagas disease). Infect Dis Clin N Am 26: 275-291.

Reuter JS, Mathews DH 2010. RNAstructure: software for RNA secondary structure prediction and analysis. BMC Bioinformatics 11: 129.

Sauvage V, Aubert D, Escotte-Binet S, Villena I 2009. The role of ATP-binding cassette (ABC) proteins in protozoan parasites. $M o l$ Biochem Parasitol 167: 81-94.

Sharom FJ 2008. ABC multidrug transporters: structure, function and role in chemoresistance. Pharmacogenomics 9: 105-127.

Siegel TN, Gunasekera K, Cross GAM, Ochsenreiter T 2011. Gene expression in Trypanosoma brucei: lessons from high-throughput RNA sequencing. Trends Parasitol 27: 434-441.

Sosa-Estani S, Viotti R, Segura EL 2009. Therapy, diagnosis and prognosis of chronic Chagas disease: insight gained in Argentina. Mem Inst Oswaldo Cruz 104 (Suppl. I): 167-180.

Torres C, Barreiro L, Dallagiovanna B, Gamarro F, Castanys S 1999. Characterization of a new ATP-binding cassette transporter in Trypanosoma cruzi associated to a L1Tc retrotransposon. Biochim Biophys Acta 1489: 428-432.

Torres C, Pérez-Victoria FJ, Parodi-Talice A, Castanys S, Gamarro F 2004. Characterization of an ABCA-like transporter involved in vesicular trafficking in the protozoan parasite Trypanosoma cruzi. Mol Microbiol 54: 632-646.
Urbina JA 2010. Specific chemotherapy of Chagas disease: relevance, current limitations and new approaches. Acta Trop 115: 55-68.

Velamakanni S, Wei SL, Janvilisri T, van Veen HW 2007. ABCG transporters: structure, substrate specificities and physiological roles: a brief overview. J Bioenerg Biomembr 239: 465-471.

Villarreal D, Barnabé C, Sereno D, Tibayrenc M 2004. Lack of correlation between in vitro susceptibility to benznidazole and phylogenetic diversity of Trypanosoma cruzi, the agent of Chagas disease. Exp Parasitol 108: 24-31

Weatherly DB, Boehlke C, Tarleton RL 2009. Chromosome level assembly of the hybrid Trypanosoma cruzi genome. BMC Genomics 10: 255.

WHO - World Health Organization 2012. Research priorities for Chagas disease, human African trypanosomiasis and leishmaniasis. Available from: apps.who.int/iris/bitstream/10665/77472/1/ WHO_TRS 975 eng.pdf.

Yanase K, Tsukahara S, Mitsuhashi J, Sugimoto Y 2006. Functional SNPs of the breast cancer resistance protein-therapeutic effects and inhibitor development. Cancer Lett 234: 73-80.

Yao Z, Weinberg Z, Ruzzo W 2006. CMfinder - a covariance model based RNA motif finding algorithm. Bioinformatics 22: 445-452.

Zingales B, Andrade SG, Briones MRS, Campbell DA, Chiari E, Fernandes O, Guhl F, Lages-Silva E, Macedo AM, Machado CR, Miles MA, Romanha AJ, Sturm NR, Tibayrenc M, Schijman AG 2009. A new consensus for Trypanosoma cruzi intraspecific nomenclature: second revision meeting recommends $\mathrm{TcI}$ to $\mathrm{TcVI}$. Mem Inst Oswaldo Cruz 104: 1051-1054.

Zingales B, Miles MA, Campbell DA, Tibayrenc M, Macedo AM, Teixeira MMG, Schijman AG, Llewelly MS, Lages-Silva E, Machado CR, Andrade SG, Sturm NR 2012. The revised Trypanosoma cruzi subspecific nomenclature: rationale, epidemiological relevance and research applications. Infect Genet Evol 12: 240-253.

Zingales B, Miles MA, Moraes CB, Luquetti A, Guhl F, Schijman AG, Ribeiro I 2014. Drug discovery for Chagas disease should consider Trypanosoma cruzi strain diversity. Mem Inst Oswaldo Cruz 109: 828-833. 